\title{
A Case of Familial Cutaneous Collagenoma
}

\author{
Mi-Youn Park, M.D., Yeon-Jin Choi, M.D., Jung-Yeon Lee, M.D., Mi-Seon Shin, M.D., Ji-Young Ahn, \\ M.D.
}

Department of Dermatology, National Medical Center, Seoul, Korea

Familial cutaneous collagenoma is a rare hereditary disease that is inherited in an autosomal dominant pattern. It is characterized by early onset of multiple, skin-colored, sometimes hypopigmented cutaneous nodules, which initially show a symmetrical arrangement on the trunk, and later on the neck and upper limbs. We report on a case of a 45-year-old female who presented with multiple oval to round hypopigmented papules measuring $5 \sim 15 \mathrm{~mm}$ on her trunk. Histopathologically, the lesions showed an increased amount of collagen fibers and decreased, fragmented elastic fibers in the dermis. The skin lesions were diagnosed as familial cutaneous collagenoma and no treatment was administered. To the best of our knowledge, our case is the first reported case of familial cutaneous collagenoma (FCC) in the Korean literature. (Ann Dermatol 23(S1) S119 S122, 2011)

-Keyword-

Collagenoma

\section{INTRODUCTION}

Familial cutaneous collagenoma (FCC) is a rare autosomal dominant condition characterized by multiple cutaneous papules and nodules, primarily appearing on the trunk after puberty. These lesions are typically asymptomatic;

Received February 17, 2010, Revised October 26, 2010, Accepted for publication October 26, 2010

Corresponding author: Ji-Young Ahn, M.D., Department of Dermatology, National Medical Center, 18-79 Euljiro 6-ga, Jung-gu, Seoul 100-799, Korea. Tel: 82-2-2260-7315, Fax: 82-2-2277-0915, E-mail: aj222@ dreamwiz.com

This is an Open Access article distributed under the terms of the Creative Commons Attribution Non-Commercial License (http:// creativecommons.org/licenses/by-nc/3.0) which permits unrestricted non-commercial use, distribution, and reproduction in any medium, provided the original work is properly cited. however, on occasion, mild pruritus may occur and potentially spread with age ${ }^{1}$.

We present clinical and histopathological findings in a case of cutaneous collagenoma that has a familial tendency.

\section{CASE REPORT}

A 45-year-old female patient visited the hospital for evaluation of multiple oval to round hypopigmented papules on her trunk and limbs. The lesions first appeared at the age of 15 years and had been slowly increasing in number and size. Dermatological examination revealed multiple oval to round hypopigmented papules measuring 5 $15 \mathrm{~mm}$ on her abdomen, chest, flanks, and back (Fig. 1). The patient's past history did not show any specific findings. According to her family history, the symptoms described above also occurred in her father, two younger brothers, older sister, and her daughter, starting in puberty. Her father had lesions on his abdomen and back, her sister on her abdomen, back, and upper extremities, her two brothers on their abdomens and backs, and her daughter on her lower abdomen (Fig. 2). The initial clinical diagnosis was collagenoma and multiple cutaneous hamartomatous neoplasm caused by Cowden's disease. Laboratory test results, including urinalysis, liver function test, and electrocardiogram, showed normal or negative findings, and no anomalies were noted on her x-ray examination (hands, feet, chest, pelvis, supernumerary vertebrae, and ribs). Laboratory evaluations for breast, thyroid, and Gl tract cancer, which may accompany Cowden's disease, were also conducted; however, no abnormal findings were revealed.

A skin biopsy specimen demonstrated increased amounts of collagen in the dermis, particularly in the reticular dermis (Fig. 3A). Masson's trichrome stain revealed thickened and irregularly arranged dense fascicular bundles of collagen (Fig. 3B). Van Gieson-stained elastic bundles were decreased and fragmented (Fig. 3C). 

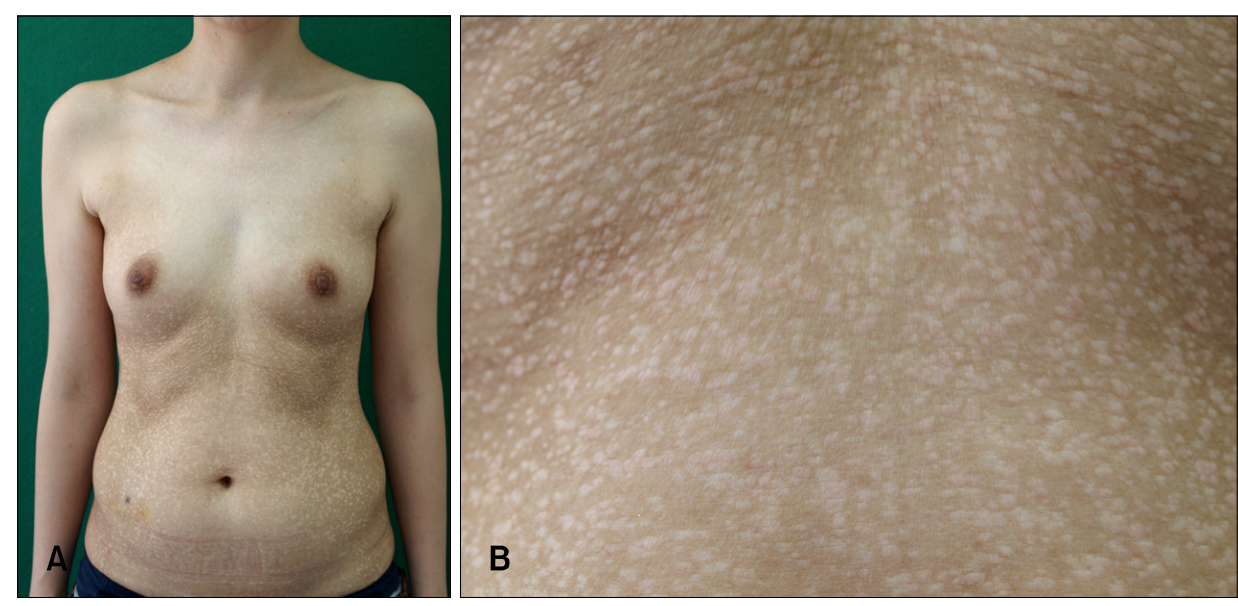

Fig. 1. (A) Multiple $5 \sim 15 \mathrm{~mm}$ sized oval to round hypopigmented papules and patches on the patient's abdomen, chest, and flank. (B) The magnified clinical feature on abdomen.

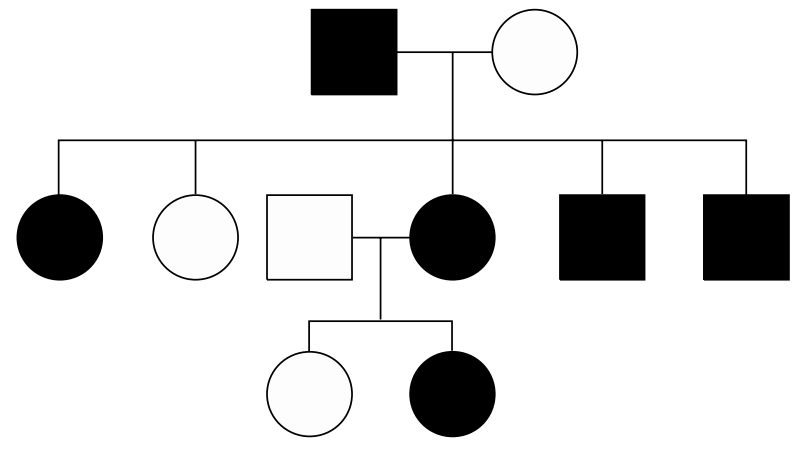

Fig. 2. Pedigree of the family. Men are represented by squares, women by circles. The solid symbols denote the cases.

On the basis of the family history and histological observations, the patients were diagnosed as having familial cutaneous collagenoma. No specific treatment was administered and the patient has been regularly followed up.

\section{DISCUSSION}

FCC was first described by Henderson et al. in $1968^{2}$. Afterward, a second case, which was hereditary, similar to the first case, was reported by Uitto et al. in $1979^{3}$, and showed a pattern of cutaneous nodules due to increased collagen deposition. From this case, FCC was shown to have autosomal dominant inheritance. Among the family members reported in the case, two patients were observed to have an increased number of lesions during pregnancy. These observations led to the hypothesis that a hormonal component may control the onset of collagenomas ${ }^{3}$. In our patient, lesions first appeared in puberty and their number showed a significant increase during pregnancy. In both previous reports, the question of an association between FCC and underlying cardiac disease was raised and was attributed to a possible fibrotic process affecting the bundle of this and other heart structures ${ }^{1,3}$. In addition, there are a number of associated conditions; however, in view of the small number of documented cases, their significance is uncertain. These include hypogonadism, congenital exophthalmos, learning disabilities, hypertrichosis, nystagmus, café-au-lait macules, and acanthosis nigricans ${ }^{2,4}$. In the present study, five of our patient's family members had similar skin findings; however, none of them had a history of cardiac disease. Our patient was healthy, with no other laboratory abnormalities and the chest $\mathrm{x}$-ray and electrocardiogram were within normal limits. Therefore, the authors did not find an association between FCC and heart disease.

The patient's cutaneous findings were consistent with those of the previous study on FCC. Asymptomatic, symmetrically distributed, cutaneous papules were located on her upper back and trunk. Typically, lesions in the second decade show a symmetrical distribution on the trunk and upper arms, with most of the nodules confined to the upper two-thirds of the back ${ }^{1-3,5,6}$. From a histologic point of view, cutaneous lesions of FCC are similar to connective tissue collagen-type nevi and to shagreen patches of tuberous sclerosis ${ }^{3}$. Uitto et al. ${ }^{1}$ have classified connective tissue nevi into three groups based on their predominant dermal component collagen, proteoglycan, and elastin, as well as their inheritance pattern.

FCC, rather than tuberous sclerosis, was diagnosed in this case due to the absence of associated findings, such as adenoma sebaceum, periungual fibromas, and ash-leaf macules $^{6}$. Another inherited, similar condition, characterized by the presence of FCC, is Buschke-Ollendorf syndrome. It is characterized by small, asymptomatic papules called disseminated dermatofibrosis lenticularis ${ }^{7,8}$. However, this syndrome is characterized by the presence 

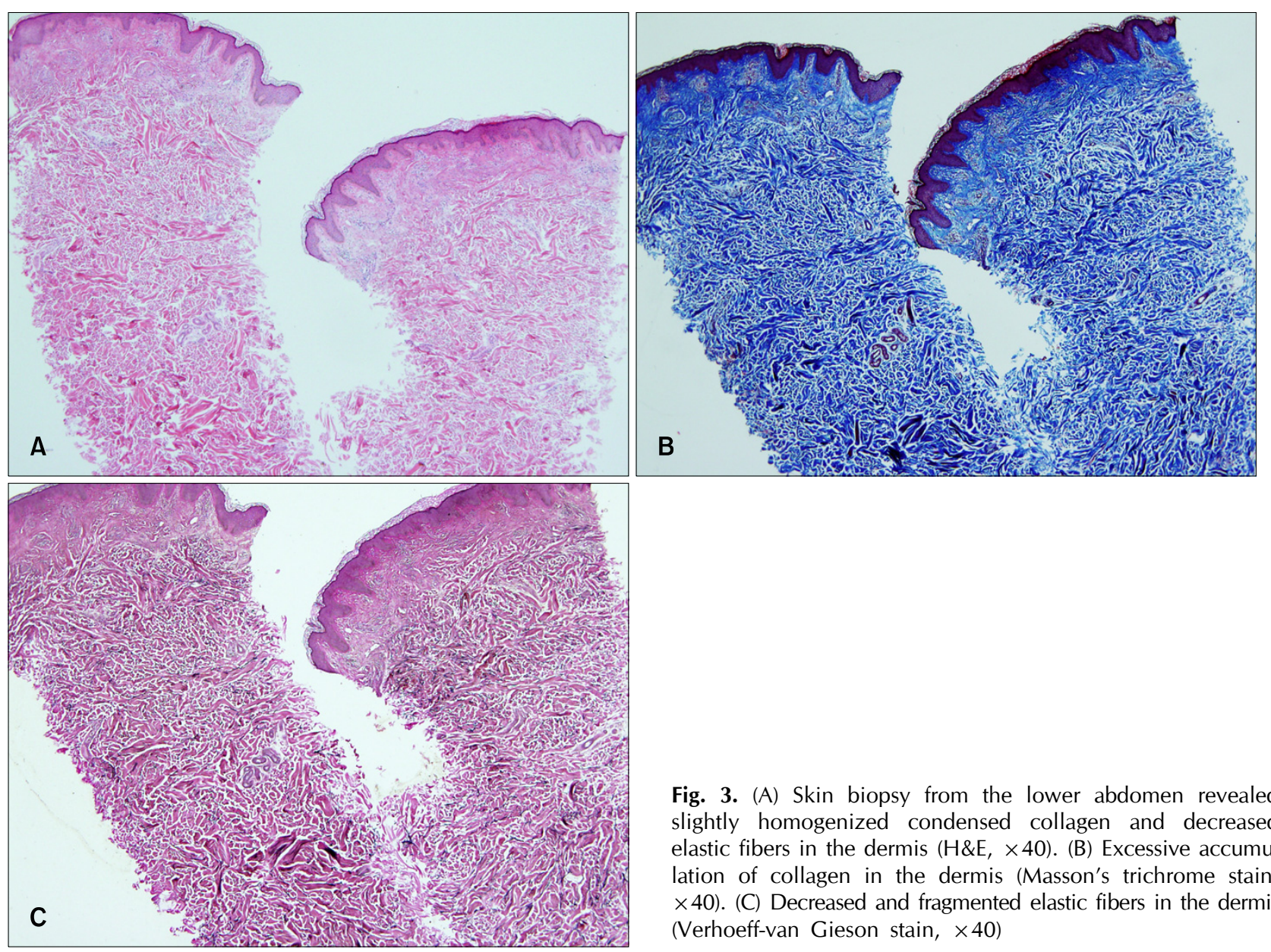

Fig. 3. (A) Skin biopsy from the lower abdomen revealed slightly homogenized condensed collagen and decreased elastic fibers in the dermis $(H \& E, \times 40)$. (B) Excessive accumulation of collagen in the dermis (Masson's trichrome stain, $\times 40$ ). (C) Decreased and fragmented elastic fibers in the dermis (Verhoeff-van Gieson stain, $\times 40$ )

of multiple cutaneous elastomas and osteopoikilosis ${ }^{9}$. In this case, no anomalies were noted on her x-ray examinations (hands, feet, chest, pelvis, supernumerary vertebrae, and ribs). She showed normal physical development, with neither internal problems nor evidence of orthopedic problems.

A skin biopsy with special stains will confirm the diagnosis by distinguishing disorders of elastin, collagen, and proteoglycan. The lesion is characterized by a markedly thickened dermis due to broad bundles of haphazardly arranged or homogenized collagen bundles ${ }^{3,5,6}$. Elastic tissue may appear to be normal or abnormal, fragmented, and diminished in quantity ${ }^{3}$.

This case needs to be differentiated from eruptive collagenoma and white fibrous papulosis of the neck (WFPN), which indicate other collagen diseases. Unlike FCC, eruptive collagenoma has no family history and no combined disorders; however, they have some common clinical features. The latter, WFPN shows multiple sharply circumscribed, round to oval shaped, white papules on the back and neck of patients with the disease. Unlike this case, these papules do not tend to coalesce, to be related

to hair follicles, or to appear on the extremities.

No successful therapeutic options have been reported, and connective tissue nevi and FCC lesions have persisted in all patients ${ }^{10}$. There is no malignant potential and most lesions do not require treatment. Surgical excision may occasionally be indicated for cosmetic reasons ${ }^{11}$.

In summary, the histologic features of the skin lesions were consistent with those of connective tissue nevi of the collagen type, and the family history led us to a diagnosis of FCC. To the best of our knowledge, our case is the first reported case of FCC in the Korean literature. Therefore, we report on this rare and interesting case of FCC. More experience with this rare condition and trials of various treatment modalities are needed.

\section{REFERENCES}

1. Uitto J, Santa Cruz DJ, Eisen AZ. Connective tissue nevi of the skin. Clinical, genetic, and histopathologic classification of hamartomas of the collagen, elastin, and proteoglycan type. J Am Acad Dermatol 1980;3:441-461.

2. Henderson RR, Wheeler CE Jr, Abele DC. Familial cutaneous 
collagenoma. Report of cases. Arch Dermatol 1968;98: 23-27.

3. Uitto J, Santa-Cruz DJ, Eisen AZ. Familial cutaneous collagenoma: genetic studies on a family. $\mathrm{Br} J$ Dermatol 1979;101:185-195.

4. Boente Mdel C, Primc NB, Asial RA, Winik BC. Familial cutaneous collagenoma: a clinicopathologic study of two new cases. Pediatr Dermatol 2004;21:33-38.

5. Sacks HN, Crawley IS, Ward JA, Fine RM. Familial cardiomyopathy, hypogonadism, and collagenoma. Ann Intern Med 1980;93:813-817.

6. Phillips JC, Knautz MA, Sangueza OP, Davis LS. Familial cutaneous collagenoma. J Am Acad Dermatol 1999;40:255257.

7. Morrison JG, Jones EW, MacDonald DM. Juvenile elastoma and osteopoikilosis (the Buschke--Ollendorff syndrome). $\mathrm{Br}$ J
Dermatol 1977;97:417-422.

8. Raque CJ, Wood MG. Connective-tissue nevus. Dermatofibrosis lenticularis disseminata with osteopoikilosis. Arch Dermatol 1970;102:390-396.

9. Dahan S, Bonafé JL, Laroche $M$, Rousseau $H$, Railhac JJ. Iconography of Buschke Ollendorff syndrome: $x$ ray computed tomography and nuclear magnetic resonance of osteopoikilosis. Ann Dermatol Venereol 1989;116:225-230.

10. McClung AA, Blumberg MA, Huttenbach Y, ColomeGrimmer MI, Raimer SS. Development of collagenomas during pregnancy. J Am Acad Dermatol 2005;53(2 Suppl 1):S150-S153.

11. Gurel MS, Mulayim MK, Ozardali I, Bitiren M. Familial cutaneous collagenoma: new affected family with prepubertal onset. J Dermatol 2007;34:477-481 